\title{
Eicosanoids in exhaled breath condensate and bronchoalveolar lavage fluid of patients with primary lung cancer
}

\author{
Maciej Ciebiada*, Paweł Górski and Adam Antczak \\ Department of Pneumology \& Allergy, Medical University of Lodz, Lodz, Poland
}

\begin{abstract}
Although eicosanoids are involved in lung carcinogenesis they were poorly investigated in exhaled breath condensate (EBC) and bronchoalveolar lavage fluid (BALf) in patients with primary lung cancer. In this study 17 patients with diagnosed non-small cell lung cancer, 10 healthy smokers and 12 healthy nonsmokers were included. The levels of cys-LTs, 8-isoprostane, LTB4 and PGE2 were measured before any treatment in the EBC of all patients and in BALf of patients with lung cancer by enzyme linked immunosorbent assay. 8-isoprostane, LTB4, cys-LTs and PGE2 were detectable in the EBC and BALf. There were no significant differences between healthy smokers and nonsmokers in concentrations of all measured mediators. Compared with both healthy controls, patients with diagnosed lung cancer displayed higher concentrations of cys-LTs $(p<0.05)$ and LTB4 ( $p<$ 0.05) in EBC. In patients with lung cancer, the mean concentrations of all measured mediators were significantly higher in BALf compared with EBC and there was a significant, positive correlation between concentration of cys-LTs, $\mathrm{LTB}_{4}$ and 8-isoprostane in BALf and their concentrations in the $\mathrm{EBC}(r=0.64, p<0.05, r=0.59, p<0.05, r=0.53, p<0.05$ respectively). Since cys-LT, LTB4 and 8-isoprostane concentrations in EBC from patients with lung cancer reflect their concentrations in BALf, they may serve as a possible non-invasive method to monitor the disease and to assess the effectiveness of therapy.
\end{abstract}

Keywords: Exhaled breath condensate, broncholaveolar lavage, lung cancer, leukotrienes, prostaglandins, early detection

\section{Introduction}

One problem with the monitoring of patients with non-small cell lung cancer (NSCLC) is the lack of specific markers that could reflect disease severity and progression. Classifications, which describe the extent of cancer spread and grading systems, are complicated and not always effective in monitoring a patient's recovery. Furthermore, there are no methods that could be used in ambulatory to predict early cancer recurrence. The collection of exhaled breath condensate (EBC) is an easy to perform, non-invasive and repeatable method that samples volatile and non-volatile substances directly from the respiratory tract $[1,2]$. The method offered

* Corresponding author: Maciej Ciebiada, Department of Pneumology and Allergy, Medical University of Lodz, Kopcińskiego Street 22, 90-153 Lodz, Poland. Tel.: +48 42678 7505; Fax: +48 42678 2129; E-mail: maciej_ciebiada@op.pl. a new insight into the pathology of airways inflammation $[1,3-5]$ however, in lung cancer the usefulness of EBC was poorly investigated.

Since in the lung cancer microenvironment, cyclooxygenase-2 (COX-2) and 5-lipooxygenase (5-LOX) are over-expressed [6,7] and oxidative stress is increased [8], we hypothesized that the end products of enzymatic (prostaglandin E2: PGE2, leukotriene B4: LTB4 and cysteinyl leukotrienes: cys-LTs) and nonenzymatic (8-isoprostane) metabolism of arachidonic acid could be detectable in EBC collected from patients with lung cancer.

The aim of this study was to assess the levels of cys-LTs, PGE2, LTB4 and 8-isoprostane in the EBC of patients with primary lung cancer and to compare with those detected in the bronchoalveolar lavage fluid (BALf) of patients diagnosed with NSCLC and those detected in EBC of healthy control. Furthermore, we wanted to investigate the relationship between levels of cys-LTs, PGE2, LTB4 and 8-isoprostane in EBC and 
their concentrations in BALf in patients suffering from primary lung cancer.

\section{Methods}

This study was approved by the Ethics Committee of the Medical University of Lodz and all subjects provided informed consent before participating. The characteristics of the study population are presented in Table 1 . In 17 included patients diagnose of lung cancer was based on the results of biopsies performed in the hospital. The histological type of the tumor was restricted to the NSCLC (planoepithelial cancer, stage I and II). All patients were considered as metastatic free individuals on the basis of imaging studies performed during routine diagnostic and before receiving any anticancer treatment. Furthermore, all patients with diagnosed cancer had peripheral lesions in the lung, allowing the bronchoalveolar lavage performance at the lesion site.

In all patients EBC was collected just prior to bronchoscopy. Patients were allowed to use short-acting inhaled $\beta_{2}$-agonists but steroids were prohibited for at least 4 weeks prior to sample collection. Exclusion criteria included: respiratory tract infection for at least 4 weeks preceding the samples collection, tuberculosis, severe heart failure, uncontrolled hypertension, autoimmunologic disease. Patients should have had at least $1 \mathrm{~L}$ of forced expiratory volume in one second (FEV1) on the day of bronchoscopy. 10 healthy smokers and 12 healthy nonsmokers were included as a control group and underwent spirometry and the EBC (due to the difficulty of obtaining consent from the Ethics Committee of Medical University of Lodz to perform bronchoscopy in healthy individuals).

\section{Collection of BALf}

Bronchoalveolar lavage was performed during the bronchoscopy according to international standards [9]. Briefly, patients were topically anesthetized with lidocaine spray applied to the nostril and the pharynx. A flexible bronchoscope (Pentax, Tokyo, Japan) was wedged to the bronchus supplying segment with the tumor. Next $100 \mathrm{ml}$ of sterile, isotonic $\mathrm{NaCl}$ was instilled into the segment and immediately aspirated by gentle suction. The mean recovery of BALf was $52.9 \pm 6.9 \%$. BALf was collected in sterile tubes, kept on ice and processed immediately in the laboratory. There BALf
Table 1

Study population

\begin{tabular}{|c|c|c|c|}
\hline & Lung cancer & $\begin{array}{c}\text { Control } \\
\text { (smokers) }\end{array}$ & $\begin{array}{c}\text { Control } \\
\text { (nonsmokers) }\end{array}$ \\
\hline Number of patients & 17 & 10 & 12 \\
\hline Age (year) & $54.9 \pm 12.0$ & $49.9 \pm 15.2$ & $37.8 \pm 13.1$ \\
\hline $\begin{array}{l}\text { Sex } \\
(\mathrm{M}: \mathrm{F})\end{array}$ & $9: 8$ & $5: 5$ & $7: 5$ \\
\hline $\begin{array}{l}\text { FEV } 1 \text { ( } \% \text { of } \\
\text { predicted value) }\end{array}$ & $77.4 \pm 9.4$ & $88.8 \pm 7.8$ & $95.6 \pm 8.9$ \\
\hline $\begin{array}{l}\text { BALf recovery } \\
(\%)\end{array}$ & $52.9 \pm 6.9$ & ND & ND \\
\hline EBC volume (ml) & $1.57 \pm 0.2$ & $1.78 \pm 0.4$ & $1.77 \pm 0.3$ \\
\hline $\begin{array}{l}\text { Smoking history } \\
\text { (years) }\end{array}$ & $16.3 \pm 4.7$ & $13.1 \pm 2.7$ & 0 \\
\hline Packyears & $20.3 \pm 6.2$ & $16.1 \pm 3.2$ & 0 \\
\hline Ex smokers (n) & 6 & 2 & 0 \\
\hline Current smokers (n) & 11 & 8 & 0 \\
\hline
\end{tabular}

Values given as the mean $\pm \mathrm{SD}, \mathrm{ND}-$ not done, $\mathrm{n}$ number of patients.

was pooled, passed through a sterile nylon filter (Becton Dickinson, NJ, USA) and centrifuged (10 min, $\mathrm{x}$ 300 , room temperature). Supernatant was collected for further analysis, whereas cell viability was performed by tryptan blue exclusion. The cells were counted under a light microscope and numbers of neutrophils, macrophages, lymphocytes and eosinophils were presented as a percentage of total cell count.

\subsection{Exhaled breath condensate collection}

In all patients EBC was collected prior to the bronchoscopy. Patients were instructed to breathe at a normal frequency and tidal volume through a mouthpiece connected to the sampling tube for 10-15 minutes with the respiratory rate between $15-20$ breaths $/ \mathrm{min}$. Patients wore a nose clip and were asked to rinse their mouth with distilled water before and after the procedure to reduce evaporation of eicosanoids from the saliva and nasal spaces. The condensate was collected by a commercially available condenser (EcoScreen, Jaeger, Germany). Samples were tested for salivary contamination by the determination of amylase activity (Sigma-Aldrich, Poznan, Poland). Approximately $1.57 \pm 0.2 \mathrm{ml}, 1.78 \pm 0.4 \mathrm{ml}$ and $1.77 \pm 0.3 \mathrm{ml}$ of condensate was collected in patients with diagnosed lung cancer, healthy smokers and non-smoking subjects respectively and immediately stored up to 4 weeks at $-80^{\circ} \mathrm{C}$ for further analysis.

\subsection{Quantification of eicosanoids in EBC and BALf}

\subsubsection{Leukotrienes}

The EBC and BALf concentration of cysteinylleukotrienes (Cys-LTs) was examined by an enzyme 
Table 2

Concentrations of cys-LTs, LTB4, PGE2 and 8-isoprostane in BALf and EBC of patients with lung cancer and healthy controls (in pg/ml)

\begin{tabular}{|c|c|c|c|c|c|c|c|c|}
\hline & \multicolumn{2}{|c|}{ cys-LTs } & \multicolumn{2}{|c|}{$\mathrm{LTB}_{4}$} & \multicolumn{2}{|c|}{$\mathrm{PGE}_{2}$} & \multicolumn{2}{|c|}{ 8-isoprostane } \\
\hline & $\overline{\text { BALf }}$ & EBC & $\overline{\text { BALf }}$ & EBC & BALf & EBC & $\overline{\text { BALf }}$ & EBC \\
\hline $\begin{array}{l}\text { Healthy } \\
\text { smokers }\end{array}$ & N.D. & $\begin{array}{c}16.88 \pm 5.3 \\
(6.91) \\
{[2.5 ;} \\
34.93]\end{array}$ & N.D. & $\begin{array}{c}16.02 \pm 4.4 \\
(12.5) \\
{[8.67 ;} \\
20.07]\end{array}$ & N.D. & $\begin{array}{c}16.6 \pm 4.1 \\
(14.99) \\
{[2.95 ;} \\
28.66]\end{array}$ & N.D. & $\begin{array}{c}15.06 \pm 5.1 \\
(10.1) \\
{[5.96 ;} \\
20.72]\end{array}$ \\
\hline $\begin{array}{l}\text { Healthy } \\
\text { nonsmokers }\end{array}$ & N.D. & $\begin{array}{c}13.73 \pm 4.6 \\
(6.8) \\
{[2.5 ;} \\
25.07]\end{array}$ & N.D. & $\begin{array}{c}12.52 \pm 2.84 \\
(10.95) \\
{[3.72 ;} \\
14.65]\end{array}$ & N.D. & $\begin{array}{c}15.63 \pm 3.85 \\
(12.37) \\
{[2.5 ;} \\
28.28]\end{array}$ & N.D. & $\begin{array}{c}12.29 \pm 3.1 \\
(10.79) \\
{[2.5 ;} \\
23.9]\end{array}$ \\
\hline $\begin{array}{l}\text { Lung } \\
\text { tumours }\end{array}$ & $\begin{array}{c}39.2 \pm 7.6 \\
(36.8) \\
{[18.45} \\
48.54]\end{array}$ & $23.4 \pm 2.7^{*}$ & $83.8 \pm 14.7$ & $23.8 \pm 2.9^{*}$ & $\begin{array}{c}27.6 \pm 5.8 \\
(17.5) \\
{[7.5 ;} \\
54.5]\end{array}$ & $\begin{array}{c}17.7 \pm 2.4 \\
(17.5) \\
{[7.5 ;} \\
24.74]\end{array}$ & $61.0 \pm 11.4$ & $18.2 \pm 3.0$ \\
\hline
\end{tabular}

Values are presented as mean \pm SEM, and (median) [25th; 75th percentile]. N.D. - not done, $* p<0.05$ (vs healthy smokers and healthy nonsmokers).

immunoassay (EIA) kit (Cayman Chemical, MI, USA) as previously described [10]. $\mathrm{LTB}_{4}$ was measured by an EIA kit (Cayman Chemical, MI, USA). The antiserum used in this assay has $100 \%$ cross-reactivity with $\mathrm{LTB}_{4}, 39 \%$ with 6-trans $\mathrm{LTB}_{4}$, and $<0.01 \%$ with $\mathrm{LTC}_{4}, \mathrm{LTE}_{4}, \mathrm{LTD}_{4}$, and $\mathrm{LTF}_{4}$. The minimum detectable concentrations were: $13 \mathrm{pg} / \mathrm{ml}$ for cys-LTs and $4.43 \mathrm{pg} / \mathrm{ml}$ for $\mathrm{LTB}_{4}[11]$.

\subsubsection{8-isoprostane}

8-isoprostane concentration in EBC and BALf was measured by the EIA kit (Cayman Chemical, MI, USA) as previously described [12]. The antiserum used in this assay has $100 \%$ cross-reactivity with 8isoprostane, $0.77 \%$ with prostaglandin F1, $0.66 \%$ with prostaglandin $\mathrm{F} 3,0.31 \%$ with prostaglandin E1. The detection limit of the assay was $5 \mathrm{pg} / \mathrm{ml}$.

\subsection{3. $P G E_{2}$}

Prostaglandin $\mathrm{E}_{2}$ concentration in $\mathrm{EBC}$ and BALf was measured by the EIA kit (Cayman Chemical, MI, USA). The antiserum used in this assay has $100 \%$ crossreactivity with $\mathrm{PGE}_{2}, 43 \%$ with $\mathrm{PGE}_{3}, 18.7 \%$ with $\mathrm{PGE}_{1}, 0.1 \%$ each with $\mathrm{PGF}_{2 \alpha}, \mathrm{PGA}_{1}, \mathrm{PGA}_{2}$. The minimum detection limit was $1.5 \mathrm{pg} / \mathrm{ml}$.

\subsubsection{Lung function test}

Pulmonary function parameters were measured before the bronchoscopy, with a computer-assisted spirometer (Lung Test 1000, Mes Dymek, Poland) according to standardized guidelines and best value of three maneuvers was expressed as a percentage of the predicted normal value.

\subsubsection{Statistical analysis}

Mean \pm standard error of the mean (SEM) was provided for normally distributed data whereas median values with 25th and 75th percentiles were also presented when the data were not normally distributed. A MannWhitney test was used to compare groups and correlations between variables were evaluated by Spearman's test. Significance was defined as a value of $p<0.05$ (GraphPad Prism 5, San Diego, CA).

\section{Results}

Three of NSCLS patients, two of healthy smokers and 6 of non-smoking healthy had 8 isoprostane below the detection limit. For LTB4 two of NSCLS patients, two of healthy smokers and three of nonsmoking healthy had concentrations below the detection limit whereas for cys-LTs concentrations below the detection limit were in two of NSCLS patients, six of healthy smokers and five of non-smoking healthy.

In patients with diagnosed lung cancer there were higher concentrations of cys-LTs $(p<0.05)$ and LTB4 $(p<0.05)$ in EBC compared to both healthy controls (Table 2). Furthermore concentrations of $\mathrm{LTB}_{4}$, 8isoprostane and cys-LTs, were significantly higher in BALf compared to EBC ( $p<0.05, p<0.001, p<$ 0.01 respectively). Mean levels of PGE2 were higher in BALf when compared to EBC however, the difference was not significant $(p>0.05)$ (Table 2, Fig. 1). In patients with lung cancer concentration of LTB4 was highest both in BALf and EBC and the concentration of PGE2 was lowest both in BALf and EBC when compared with other mediators (Table 2, Fig. 1). 


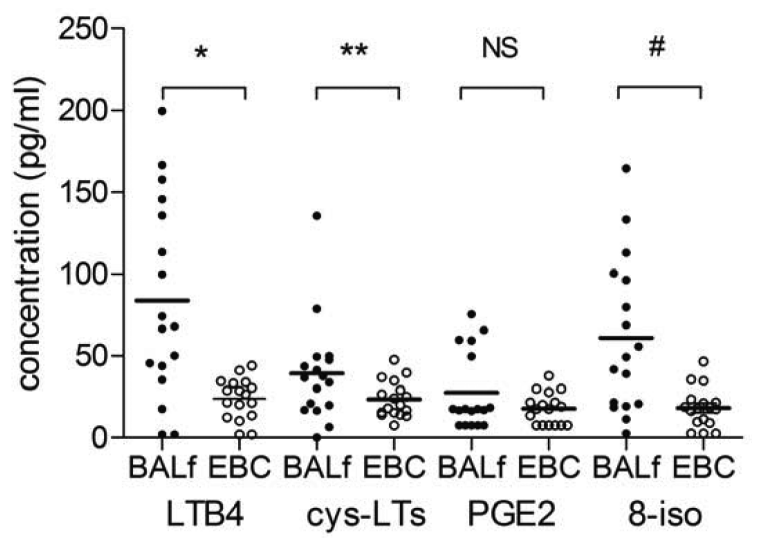

Fig. 1. Comparison of LTB4, cys-LTs, PGE-2 and 8-isoprostane in the BALf and EBC of patients with NSCLC. $* p<0.001$, ** $p<$ 0.05 , \# $p<0.01$, NS - not significant.

Table 3

Correlation between mediators in EBC or BALf with BALf cytology in patients with NSCLC

\begin{tabular}{lllc}
\hline & & \multicolumn{2}{c}{ Correlations A with B } \\
\cline { 3 - 4 } Variable A & Variable B & \multicolumn{1}{c}{$\mathrm{p}$} & $\mathrm{p}$ value \\
\hline cys-LTs in EBC & macrophages in BALf & -0.67 & 0.003 \\
cys-LTs in BALf & basophils in BALf & -0.49 & 0.04 \\
PGE2 in BALf & eosinophils in BALf & -0.6 & 0.01 \\
PGE2 in BALf & basophils in BALf & -0.56 & 0.02 \\
\hline
\end{tabular}

There was a strong positive correlation between both cys-LTs, $\mathrm{LTB}_{4}$ and 8-isoprostane in BALf and those measured in the EBC $(r=0.64, p<0.05, r=0.59$, $p<0.05, r=0.53 p<0.05$ respectively) (Fig. 2) whereas correlation for PGE2 was not significant $(r=$ $0.47, p>0.05)$. We did not find correlations between the values of FEV1 and concentrations of eicosanoids both in EBC and BALf.

The cellular profile of BALf in patients with diagnosed lung cancer was as follows: macrophages $85.2 \pm$ $1.8 \%$, lymphocytes $9.8 \pm 1.8 \%$, neutrophils $1.8 \pm$ $0.5 \%$, monocytes $0.9 \pm 0.3 \%$, eosinophils $1.1 \pm 0.3 \%$, basophils $0.6 \pm 0.2 \%$, epithelium $0.3 \pm 0.1 \%$, vitality $98.3 \pm 0.2 \%$. There were some correlations between cell counts in BALf and mediators measured in both BALf and EBC. Briefly, the values of PGE2 in the BALf of patients with lung cancer presented a significant negative correlation with BALf basophils and eosinophils levels, cys-LTs in the BALf presented a significant negative correlation with BALf basophils levels whereas cys-LTs in EBC correlated negatively and significantly with BALf macrophages count (Table 3).

Although levels of LTB4, cys-LTs, PGE2 and 8isoprostane were generally lower in EBC of healthy nonsmokers no statistical differences were found be- tween the healthy smoking and nonsmoking subjects (Table 2).

\section{Discussion}

Our study demonstrates that 8-isoprostane, LTB4, cys-LTs and PGE2 are detectable in EBC and their concentrations are significantly lower than those in BALf in patients with diagnosed NSCLC. The concentrations of LTB4 and cys-LTs in breath condensate of these patients were higher than the concentrations measured in $\mathrm{EBC}$ of healthy, age-matched smokers and healthy nonsmokers. We demonstrated, for the first time, significant, positive correlations between levels of exhaled cys-LTs, LTB4 and 8-isoprostane and those of BALf and the lack of correlation between levels of these mediators in BALf and EBC and lung function parameters.

Breath condensate may provides information about pulmonary inflammation and oxidative stress. Collection of EBC has several advantages over the traditional sampling from the airways with BAL. It is easy to perform, non-invasive, repeatable, standardized [13] and collects metabolites originating locally in the airways and lungs [1,2]. Various gases and non-volatile compounds have been detected and studied in the EBC that may reflect the concentrations within the extracellular epithelial lining fluid in the airways. Biomarkers found in EBC were used to monitor asthma [1,2], COPD [1] and other inflammatory diseases $[4,14]$ while in patients with lung cancer were poorly investigated.

It was demonstrated that lung carcinogenesis is associated with overexpression of COX-2 and 5-LOX [6, 7,15] and enhanced oxidative stress [16]. Their end products (LTB4, cys-LTs, PGE2, 8-isoprostane) are increased in the airway lumen of patients with lung cancer and have been implicated in peritumoral inflammation [14,16-19], tumor development [7,20-22], angiogenesis $[23,24]$ and suppressesion of host defence mechanisms $[20,24]$.

In patients with diagnosed lung cancer levels of LTB4 were elevated in EBC [11] and pleural effusion [25] whereas 8-isoprostane was found in $\mathrm{EBC}$ and serum [16] and PGE2 was measured in EBC [22].

Similarly, we have demonstrated that the levels of 8isoprostane, LTB4 and cys-LTs are detectable in EBC. To validate the data obtained from EBC, BALf has been used in our study. Since both primary lung cancer and pulmonary metastases from other organs increase eicosanoids concentration in BALf [22] we included 

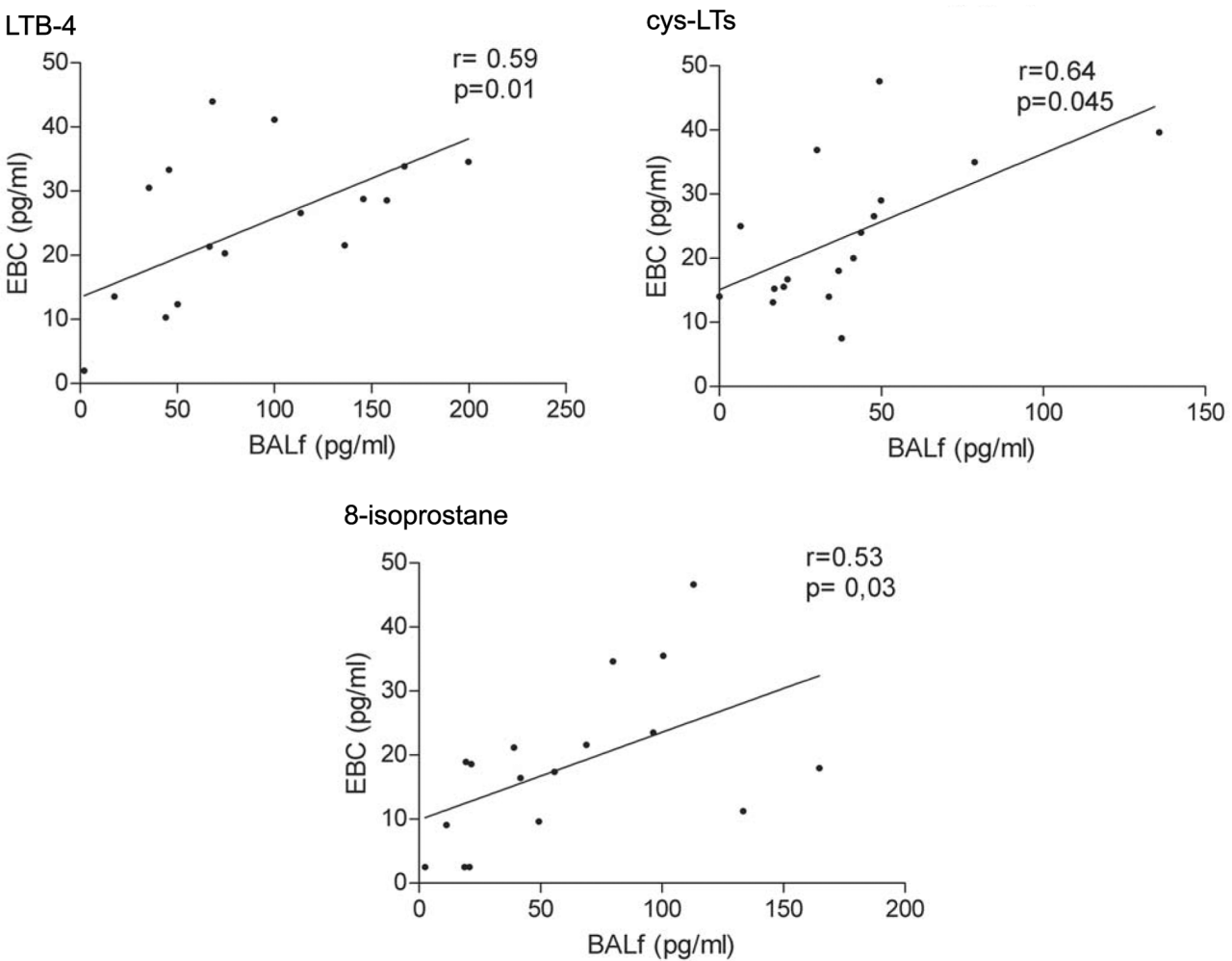

Fig. 2. Positive correlation between LTB4, cys-LTs and 8-isoprostane levels in BALf and EBC of patients with NSCLC.

patients with primary lung cancer without extrathoracic manifestations and we excluded all patients with extrathoracic malignancies that could give pulmonary metastases. Furthermore, the patients' age [19] and smoking status [26] may influence the concentrations of lipid mediators thus to eliminate the bias we have included age-matched healthy smokers and healthy, nonsmoking subjects as a control groups. In this way lipid mediators found in BALf and EBC reflected mainly its production within the lung by neoplasmatic and inflammatory milieu.

As we have demonstrated previously, the levels of PGE2 and cys-LTs were higher in EBC of patients with lung cancer when we compared with patients with noneoplasmatic diseases (COPD, chronic cough, sarcoidosis) and levels of LTB4 were higher than those observed in patients with chronic cough and sarcoidosis [27]. In this study, levels of cys-LTs and LTB4 in EBC were higher than in healthy. The explanation of this phenomenon could be twofold. Lung carcinogenesis may induce local inflammation that recruits inflammatory cells capable of releasing of reactive oxygene species and eicosanoids. However, cancer cells may induce oxidative stress and overexpress oxygenas- es synthesizing lipids mediators. In this study, similarly to other authors $[1,28]$ we did not find a significant positive correlation between the levels of eicosanoids with number of macrophages and neutrophils in BALf. Moreover, we found significant negative correlation between PGE2 and macrophages count in BALf which constitute a main source of these mediators. Therefore, similarly to other investigators $[1,28]$ we had a problem ascertaining the cellular source of mediators. However, if cancer cells are able to secrete lipids mediators [21] and there was no positive correlation between cells count and levels of lipids mediators in BALf and EBC, we may come to the plausible conclusion that tumor cells were the main source of these mediators.

Furthermore, we have demonstrated, for the first time, that concentrations of LTB4, cys-Lts and 8isporostane in EBC correlated positively with that in BALf. BALf is a sample from only a portion of the lung and does not capture mediators from the airways, whereas EBC represents a whole lung sample and contains mediators both from the affected area of the lung and the rest of respiratory truct. Therefore, the concentration of lipids mediators is the highest in bronchus supplying the lobe with the lung cancer [21] and is 
much smaller in EBC due to the significant dilution of the sample [22,29]. Nevertheless, although concentration of cys-LTs were higher in BALf, Akiyama found significant positive correlation with cys-LTs levels in EBC of patients with idiopathic pulmonary fibrosis [29] whereas Piotrowski demonstrated such positive correlation for 8-isoprostane and LTB4 in patients with sarcoidosis [4]. Similarly, in our study, the concentration of LTB4, cys-LTs and 8-isporostane may reflect their concentration in BALf in patients with diagnosed NSCLC. This finding may depend on the homogeneity of patients' groups in our study and on the higher concentrations of lipids mediators than observed previously in patients with noneoplasmatic diseases. Since different types of cancer secrete different amounts of lipids mediators [21] and extrathoracic malignances as well as metastatic tumor in the lungs from other localizations may affect eicosanoids concentration in BALf [22], we have included exclusively metastatic free patients with planoepithelial cancer.

In this study we did not follow-up our patients with expired biomarkers and we are not aware of the changes of these markers during the course of the disease and treatment. However, Funahashi [22] observed PGE2 decrease in BALf of patients with NSCLC after the successful cancer removal. This topic could be addressed in future studies.

\section{Conclusion}

The present study describes, for the first time, the quantitative assessment of cys-LTs, LTB4, PGE2 and 8-isoprostane measured simultaneously both in EBC and BALF of patients with diagnosed NSCLC. We have demonstrated that in patients with lung cancer with similar smoking habits to healthy smokers, the differences observed in LTB4 and cys-LTs are related mainly to the underlying neoplasmatic disease. As we have found a significant and positive correlation between the levels of LTB4, cys-LTs, and 8-isoprostane in EBC and BALf, we speculate that breath based detection of lipids mediators may help in early detection and monitoring of lung cancer and cancer recurrence.

\section{Acknowledgments}

This study was supported from Medical University of Lodz.

\section{Conflict of interest statement}

All authors declare no conflict of interest.

\section{References}

[1] K. Kostikas, M. Gaga, G. Papatheodorou, T. Karamanis, D. Orphanidou, S. Loukides, Leukotriene B4 in exhaled breath condensate and sputum supernatant in patients with COPD and asthma, Chest 127 (2005), 1553-1559.

[2] G.E. Carpagnano, P.J. Barnes, J. Francis, N. Wilson, A. Bush, S.A. Kharitonov, Breath condensate $\mathrm{pH}$ in children with cystic fibrosis and asthma: a new noninvasive marker of airway inflammation? Chest 125 (2004), 2005-2010.

[3] S. Carraro, B. Andreola, R. Alinovi, M. Corradi, L. Freo, L. Da Dalt, E. Baraldi, Exhaled leukotriene B4 in children with community acquired pneumonia, Pediatr Pulmonol 43 (2008), 982-986.

[4] W.J. Piotrowski, A. Antczak, J. Marczak, A. Nawrocka, Z. Kurmanowska, P. Górski, Eicosanoids in exhaled breath condensate and BAL fluid of patients with sarcoidosis, Chest 132 (2007), 589-596.

[5] G.E. Carpagnano, S.A. Kharitonov, O. Resta, M.P. FoschinoBarbaro, E. Gramiccioni, P.J. Barnes, 8-Isoprostane, a marker of oxidative stress, is increased in exhaled breath condensate of patients with obstructive sleep apnea after night and is reduced by continuous positive airway pressure therapy, Chest 124 (2003), 1386-1392.

[6] G.E. Carpagnano, A. Spanevello, G.P. Palladino, C. Gramiccioni, C. Ruggieri, F. Carpagnano, M.P. Foschino Barbaro, Cigarette smoke and increased COX-2 and survivin levels in exhaled breath condensate of lung cancer patients: how hot is the link? Lung Cancer 67 (2010), 108-113.

[7] M. Luo, S. Lee, T.G. Brock, Leukotriene synthesis by epithelial cells, Histol Histopathol 18 (2003), 587-595.

[8] A. Katsabeki-Katsafli, T. Kerenidi, K. Kostikas, E. Dalaveris, T.S. Kiropoulos, E. Gogou, A.I. Papaioannou, K.I. Gourgoulianis, Serum vascular endothelial growth factor is related to systemic oxidative stress in patients with lung cancer, Lung Cancer 60 (2008), 271-276.

[9] British Thoracic Society Bronchoscopy Guidlines Committee, a Subcommittee of the Standards of Care Committee of the British Thoracic Society, British Thoracic Society guidelines on diagnostic flexible bronchoscopy, Thorax 56 (2001), 1-21.

[10] A. Antczak, P. Montuschi, S.A. Kharitonov, P. Gorski, P.J. Barnes, Increased exhaled cysteinyl-leukotrienes and 8isoprostane in aspirin-induced asthma, Am J Respir Crit Care Med 166 (2002), 301-306.

[11] P. Montuschi, E. Ragazzoni, E. Valente, G. Corbo, C. Mondino, G. Ciappi, P.J. Barnes, G. Ciabattoni, Validation of leukotriene B4 measurements in exhaled breath condensate, Inflamm Res 52 (2003), 69-73.

[12] P. Montuschi, M. Corradi, G. Ciabattoni, J. Nightingale, S.A. Kharitonov, P.J. Barnes, Increased 8-isoprostane, a marker of oxidative stress, in exhaled condensate of asthma patients, Am J Respir Crit Care Med 160 (1999), 216-220.

[13] P.E. Silkoff, S.C. Erzurum, J.O. Lundberg, S.C. George, N. Marczin, J.F. Hunt, R. Effros, I. Horvath, ATS Workshop proceedings: exhaled nitric oxide and nitric oxide oxidative metabolism in exhaled breath condensate, Proc Am Thorac Soc 3 (2006), 131-145. 
[14] S.K. Huang, M. Peters-Golden, Eicosanoid lipid mediators in fibrotic lung diseases: ready for prime time? Chest 133 (2008), 1442-1450.

[15] S.H. Hong, I. Avis, M.D. Vos, A. Martinez, A.M. Treston, J.L. Mulshine, Relationship of arachidonic acid metabolizing enzyme expression in epithelial cancer cell lines to the growth effect of selective biochemical inhibitors, Cancer Res 59 (1999), 2223-2228.

[16] E. Dalaveris, T. Kerenidi, A. Katsabeki-Katsafli, T. Kiropoulos, K. Tanou, K. Gourgoulianis, K. Kostikas, VEGF, TNFalpha and 8-isoprostane levels in exhaled breath condensate and serum of patients with lung cancer, Lung Cancer 64 (2009), 219-225.

[17] P. Montuschi, P.J. Barnes, Exhaled leukotrienes and prostaglandins in asthma, J Allergy Clin Immunol 109 (2002), 615-620.

[18] M. Takano, H. Nishimura, Y. Kimura, J. Washizu, Y. Mokuno, Y. Nimura, Y. Yoshikai, Prostaglandin E2 protects against liver injury after Escherichia coli infection but hampers the resolution of the infection in mice, J Immunol 161 (1998), 3019-3025.

[19] M.J. Cruz, S. Sánchez-Vidaurre, P.V. Romero, F. Morell, X. Munoz, Impact of age on $\mathrm{pH}, 8$-isoprostane, and nitrogen oxides in exhaled breath condensate, Chest 135 (2009), 462467.

[20] R.M. Breyer, C.K. Bagdassarian, S.A. Myers, M.D. Breyer, Prostanoid receptors: subtypes and signaling, Annu Rev Pharmacol Toxicol 41 (2001), 661-690.

[21] A. leFever, A. Funahashi, Elevated prostaglandin E2 levels in broncholalveolar lavage fluid of patients with bronchogenic carcinoma, Chest 98 (1990), 1397-1402.

[22] A. Funahashi, R.W. Harland, A. LeFever, Association of increased prostaglandin E2 content in bronchoalveolar lavage fluid and intrathoracic malignancy, Chest 106 (1994), 166172.
[23] G. Modat, A. Muller, A. Mary, C. Gregoire, C. Bonne, Differential effects of leukotrienes $\mathrm{B}_{4}$ and $\mathrm{C}_{4}$ on bovine aortic endothelial cell proliferation in vitro, Prostaglandins 33 (1987), 531-538.

[24] H. Amano, I. Hayashi, H. Endo, H. Kitasato, S. Yamashina, T. Maruyama, M. Kobayashi, K. Satoh, M. Narita, Y. Sugimoto, T. Murata, H. Yoshimura, S. Narumiya, M. Majima, Host prostaglandin E(2)-EP3 signaling regulates tumor-associated angiogenesis and tumor growth, J Exp Med 197 (2003), 221232.

[25] E. Pace, M. Profita, M. Melis, A. Bonanno, A. Paternò, C.H. Mody, M. Spatafora, M. Ferraro, L. Siena, A.M. Vignola, G. Bonsignore, M. Gjomarkaj, LTB4 is present in exudative pleural effusions and contributes actively to neutrophil recruitment in the inflamed pleural space, Clin Exp Immunol 135 (2004), 519-527.

[26] A.J. Duffield-Lillico, J.O. Boyle, X.K. Zhou, A. Ghosh, G.S. Butala, K. Subbaramaiah, R.A. Newman, J.D. Morrow, G.L. Milne, A.J. Dannenberg, Levels of prostaglandin E metabolite and leukotriene E(4) are increased in the urine of smokers: evidence that celecoxib shunts arachidonic acid into the 5lipoxygenase pathway, Cancer Prev Res 2 (2009), 322-332.

[27] A. Antczak, W.J. Piotrowski, J. Marczak, M. Ciebiada, P. Gorski, P.J. Barnes, Correlation between eicosanoids in bronchoalveolar lavage fluid and in exhaled breath condensate, Dis Markers 30 (2011), 213-220.

[28] K. Kostikas, G. Papatheodorou, K. Psathakis, P. Panagou, S. Loukides, Oxidative stress in expired breath condensate of patients with copd, Chest 124 (2003), 1373-1380.

[29] E. Ono, H. Mita, M. Taniguchi, N. Higashi, T. Tsuburai, E. Miyazaki, T. Kumamoto, K. Akiyama, Comparison of cysteinyl leukotriene concentrations between exhaled breath condensate and bronchoalveolar lavage fluid, Clin Exp Allergy 38 (2008), 1866-1874. 


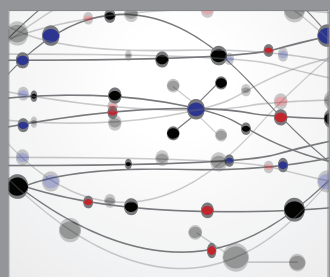

The Scientific World Journal
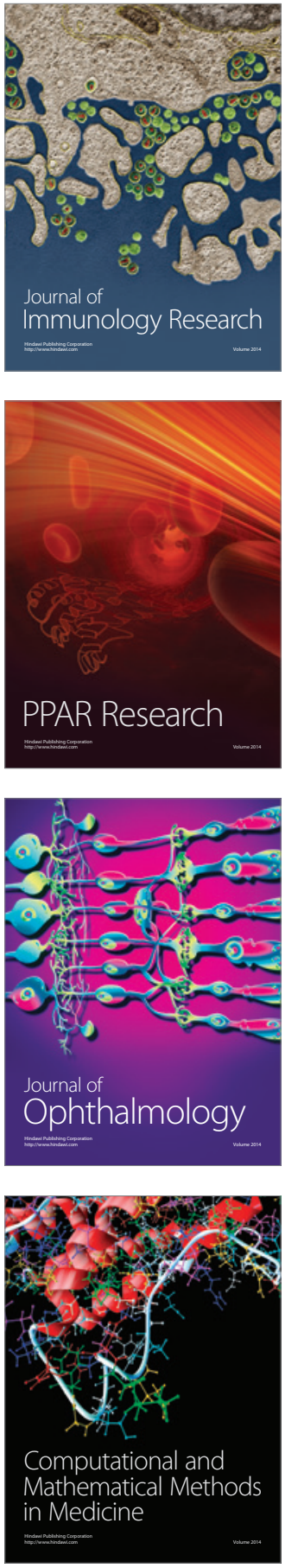

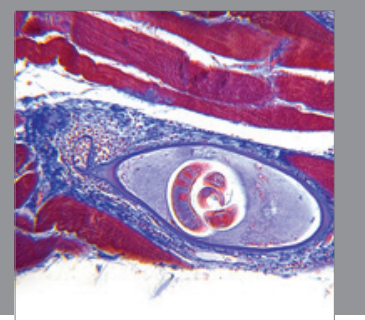

Gastroenterology

Research and Practice
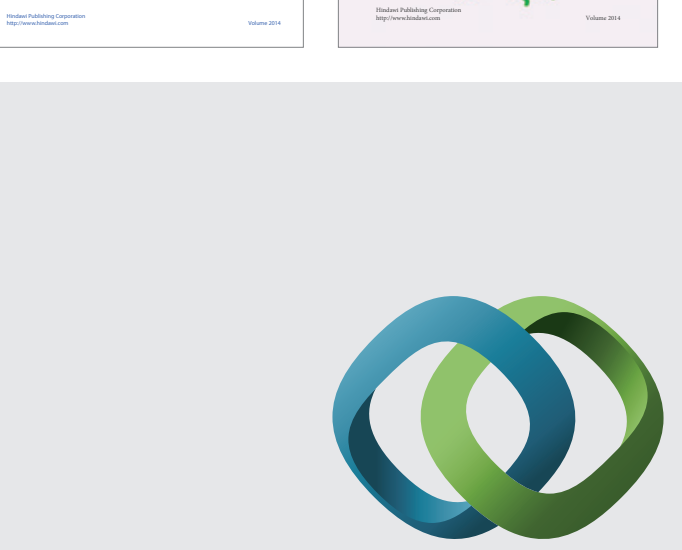

\section{Hindawi}

Submit your manuscripts at

http://www.hindawi.com
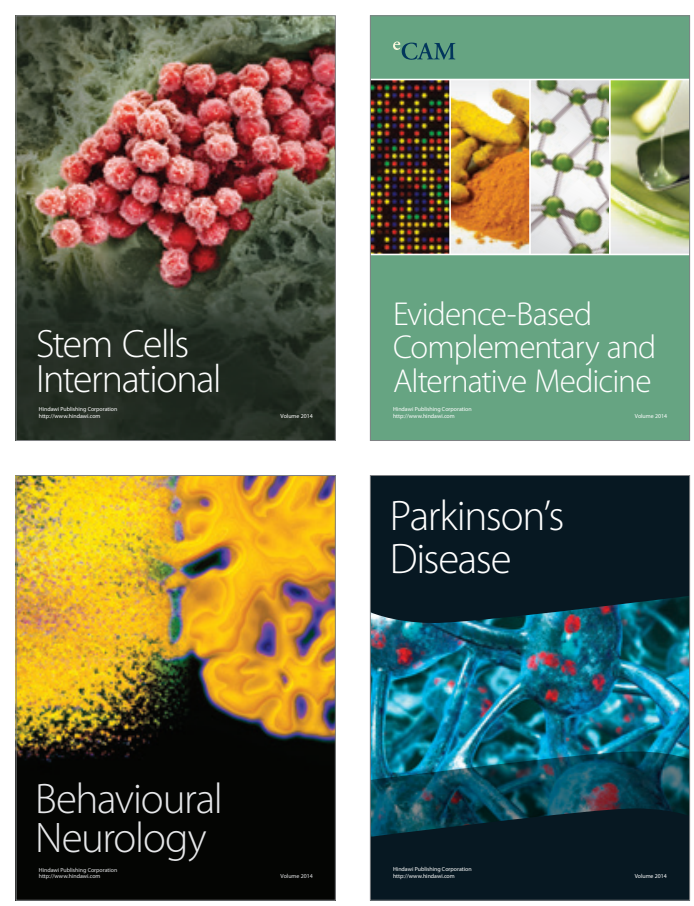

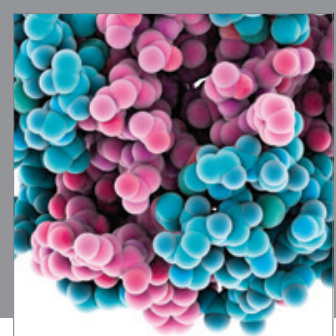

Journal of
Diabetes Research

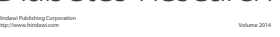

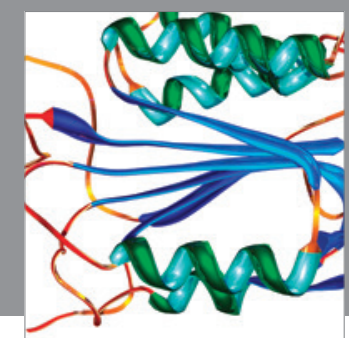

Disease Markers
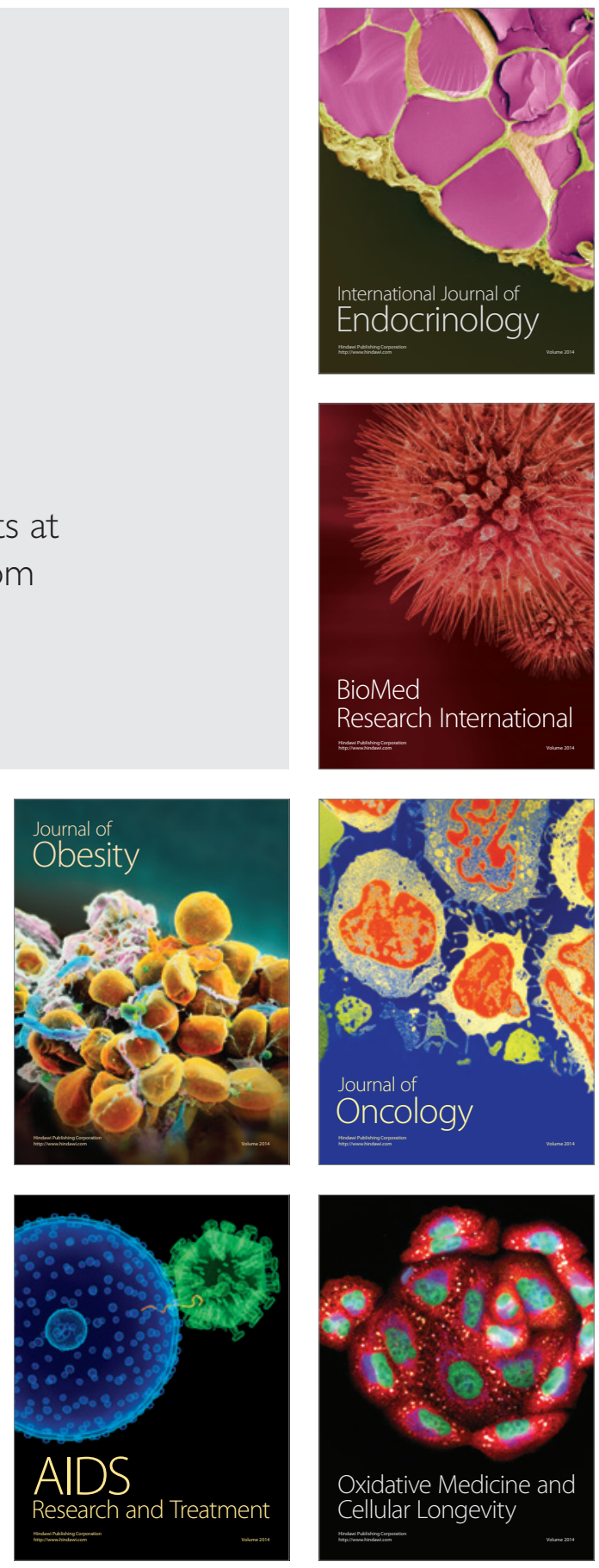